\title{
STUDIES ON THE RAPID METHODS FOR EVALUATING SEED VIGOR OF SWEET CORN
}

Guangwu Zhao ${ }^{1, *}$, Linlin Yang ${ }^{2}$, Jianhua Wang ${ }^{3}$, Zhujun Zhu ${ }^{1}$

${ }^{1}$ School of Agriculture and Food Science, Zhejiang Forestry College, Lin'an, China, 311300

${ }^{2}$ College of Foreign Languages, Wenzhou University, Wenzhou, China, 325027

${ }^{3}$ Department of Seed Science and Technology, China Agricultural University, Beijing, China, 100094;

* Corresponding author, Address: School of Agriculture and Food Science, Zhejiang Forestry College, 88 Huancheng North Road, Lin'an, 311300, P. R. China, Tel: +86-571-63742087, Fax:+86-571-63741276,Email:gwuzhao@126.com

Abstract: $\quad$ Since the germination test is still the main method for evaluating seed vigor of sweet corn, it is necessary to study the rapid methods for evaluating their vigor. First, two vigor levels of 9 super sweet corn and 9 sugar enhanced corn hybrids were distinguished by accelerated ageing treatment. Next, their vigor statuses were tested by rapid methods such as electrical conductivity (EC), soluble sugar (SS), volatile aldehyde (VA), dehydrogenase activity (DA) and Q2 oxygen sensing technology. Correlation relationship was analyzed between the above tested values and vigor performances. The results showed that EC, SS, VA, DA, oxygen metabolism rate (OMR), critical oxygen pressure (COP) were all suitable to evaluate seed vigor of sweet corn. Furthermore, VA was optimal for evaluating seed vigor of super sweet corn. DA was optimal for evaluating sugar enhanced corn.

Keywords: super sweet corn, sugar enhanced corn, seed vigor, rapid evaluation methods

\section{INTRODUCTION}

Sweet corn (Zea mays L.), especially super sweet corn and sugar enhanced corn are widely planted. Seed vigor is a comprehensive characteristic that is an important index of seed quality and is closely correlated with field performances. Field emergence percentage (FEP) and field emergence speed

Please use the following format when citing this chapter:

Zhao, G., Yang, L., Wang, J. and Zhu, Z., 2009, in IFIP International Federation for Information Processing, Volume 295, Computer and Computing Technologies in Agriculture II, Volume 3, eds. D. Li, Z. Chunjiang, (Boston: Springer), pp. 1729-1738. 
(FES) are the most direct indicators of seed vigor. FEP is the only index in most reports about seed vigor evaluation, whereas FES has been often ignored (Ching et al., 1977; Waters and Blanchette 1983; Wilson et al., 1992; Zhang 1997; Woltz and TeKrony 2001; Wang et al., 2004; Zhao and Wang 2005; Zhao et al., 2007). In our study, FEP and FES or laboratory germination percentage (LGP) and laboratory germination speed (LGS) were together considered.

The germination test is still the main method for evaluating seed vigor of sweet corn. Germination test is time consuming. Too often seed lots are sent either to the next step in processing or to the client without having been thoroughly analyzed. Due to time constraints and a competitive market, too many times seeds are processed without adequate quality information needed to get the best from the seeds. Therefore, it is necessary to study the rapid methods for evaluating their seed vigor.

Some tests such as electrical conductivity (EC), soluble sugar (SS), volatile aldehyde (VA) and dehydrogenase activity (DA) are faster than the germination test. EC can indirectly determine seed membrane integrity of different quality seed lots and is an easy, fast and objective vigor testing method. It has been listed in the International Rules for Seed Testing as a seed vigor testing method for pea. Sweet corn is famous for high sugar content. SS in the soaked solution has not been reported for evaluating seed vigor of sweet corn. In addition, VA is toxic to germinating seeds. VA released during seed germination was negatively correlated with seed vigor, which was validated in vigor testing of soybean, rice and wheat (Wilson and McDonald, 1986; Chen et al., 1996; Zhu and Zeng, 1999). It might be a potential method for vigor testing (Tao and Zheng, 1991). DA could be used to evaluate seed vigor of rice and maize (Akram et al., 2002; Qiao et al., 2003).

The Q2 oxygen sensing technology is revolutionary for seed testing in basic research and commercial operations alike. It provides a fast and accurate measurement of the germination level of a seed lot. In addition, Q2 data is more robust and defining than traditional germination test. Depending on the species, the estimated time needed will be between 5 and 96 hours and will easily determine dead, dormant or actively germinating seeds. Although it currently does not provide specific details on seedling abnormalities, the Q2 can quickly give more accurate indications of the vigor and the homogeneity of a seed lot.

The Q2 instrument, developed by Netherlands ASTEC Global Corporation, measures the percentage of oxygen in closed microtiter plates, consisting of 24-48-96 wells, in regular time intervals. The purpose of the measurements is to relate the oxygen consumption of seeds over time, to the quality of the seed lot. Q2 stands for Quality and Quick, as well as O2 (oxygen), the element the Q2 instrument is based upon. The Q2 instrument can provide results far more quickly than a germination test. Moreover, due 
to the latest developments in optical technology for measuring oxygen, the Q2 instrument is able to detect seeds' activity in the first stages of germination (imbibition). Imbibition is characterized by an increased amount of oxygen consumption. In addition to very fast detection of germinating seeds, the Q2 instrument provides data concerning the dormancy of nongerminating seeds, the homogeneity of a seed lot, as well as information concerning different stress related factors, and so much more.

Q2 test can be used as fast and accurate indications of germination under certain conditions. They can be used to detect maximum germination (correlating to an International Seed Testing Association (ISTA) test) under various environmental conditions, such as temperature, moisture, and substrates. In the second case, Q2 test can be used as a stress test, telling something about the vigor of seeds. Certain abnormalities may be detected if oxygen consumption is affected. The accuracy of this test depends on the protocols used and of course, the integrity of the sample. The instrument enables testing of a large number of samples (from 1 seed to several microtiter plates) and the duration of the test will influence the quality of the results (from a minimum of a single scan to an indefinite period; 48 hours for the most common seeds). The development of protocols for specific seed testing objectives is important and the precision of executing these protocols, such as the application of water, or the substrate application, will obviously influence the results as well.

The key innovation to the Q2 oxygen sensing technology is that oxygen consumption is directly and proportionately related to energy use. Thus, a seed's energetic potential can be determined by measuring its oxygen consumption in a simulated field environment. Because of this proportional relationship between oxygen consumption (respiration) and caloric energy consumption (metabolism), robust inferences for a seed's performance in the field can be made. To characterize a greater population or seed lot, measuring respiration and metabolism seed by seed is thus very precise and accurate using the Q2 instrument. Measuring energy use can give us new insights on germination and vigor in one single, quality controlled test, quickly. Up to now, Q2 test is only successfully applied to evaluate seed vigor of a few crops such as sugar beet. Q2 test depends on the specific protocol according to specific species. Therefore, the Q2 oxygen sensing technology provides a great room to seed vigor testing of so many species.

However, whether these vigor indices are suitable for evaluating seed vigor of sweet corn needs further study. As for the Q2 oxygen sensing technology, we will focus on the initial research in super sweet corn "Green Superman". First, the specific protocol for sweet corn is developed. Then, oxygen percentage was tested at an interval of $30 \mathrm{~min}$ by Q2 instrument. Next, five Q2 values were calculated including increased metabolism time (IMT), oxygen metabolism rate (OMR), critical oxygen pressure (COP), 
relative germination time (RGT), homogeneity, or uniformity of the RGT (HOM) developed by ASTEC. Finally, correlation relationship was analyzed between the five Q2 values and laboratory germination performances and optimal indices were confirmed. The focus of our study was to develop the rapid methods for evaluating seed vigor of sweet corn.

\section{MATERIALS AND METHODS}

\subsection{Seed materials}

Seeds of 18 sweet corn hybrids were collected from 9 provinces in China. Seeds of super sweet corn "Chaotian 204", "Chaotian 43", "Green Superman", "Nongtian 2", "Nongtian 3", "Huatian 1", "Xiangtian 1", "Yangtian 1", "Mitian 8" were bought from Zhejiang, Beijing, Guangdong, Hubei, Hunan, Jiangsu. Seeds of sugar enhanced corn "Jingketian 115", "Jingketian 116", "Tiandan 8", "Tiandan 10", "Zhongtian 2", "Nongtian 1", "Yuetian 3", "Sutian 8", "Jiatian 16" were bought from Beijing, Guangdong, Jiangsu, Shanghai. Except for 10 lots of "Green Superman" seeds, the other hybrid seeds were only bought for 1 lot.

\subsection{Accelerated ageing treatment}

To distinguish seed vigor to two levels (high vigor and low vigor), all seeds were aged in a container with $100 \%$ relative humidity at $45^{\circ} \mathrm{C}$ for $48 \mathrm{~h}$. Then, seeds were re-dried.

\subsection{Electrical conductivity test}

The EC test was conducted on three replicates of 50 seeds each for each treatment. The test referred to the international rules for seed testing (ISTA, 2007). EC was tested by a DDS-11A conductivity meter (Shanghai, China) and was calculated by the formula of the international rules for seed testing.

\subsection{Soluble sugar test}

The imbibition solution $(1 \mathrm{~mL})$ from the EC test was added to $5 \mathrm{~mL}$ anthrone, then vibrated in a test tube to mix them thoroughly. After the test tubes were stoppered, they were placed in boiling water for $10 \mathrm{~min}$ and then cooled on a shelf. The solutions were poured in colorimetric cups to 
determine the $\mathrm{OD}$ value at $620 \mathrm{~nm}$. The test was conducted on three replicates.

\subsection{Volatile aldehyde test}

Two layers of adequately moistened filter paper were placed on the bottom of a $1000 \mathrm{~mL}$ flask and 100 seeds per treatment planted on the paper until germination occurred. Then, a test tube with $5 \mathrm{~mL} 0.2 \% 3$-methyl-2benzothiazolinone hydrazone hydrochloride (MBTH) was put in the flask to imbibe VA released from the seeds. Next the orifice was stoppered and the flask was put into a case at $20^{\circ} \mathrm{C}$ in the dark. The imbibition solution $(1 \mathrm{~mL})$ was taken out after $48 \mathrm{~h}$, blended with $2.5 \mathrm{~mL} 0.2 \% \mathrm{FeCl} 3$ and reacted for 5 min. Finally, $6.5 \mathrm{~mL}$ acetone was mixed with the solution and the OD value determined at $635 \mathrm{~nm}$. $1 \mathrm{~mL} 0.2 \% \mathrm{MBTH}$ was used for the control colorimetric solution. The test was conducted on three replicates.

\subsection{Dehydrogenase activity test}

Ten embryos were extracted from seeds germinated for $1 \mathrm{~d}$ and placed into a test tube with $10 \mathrm{~mL} 0.1 \%$ triphenyl tetrazolium chloride (TTC) and the tube stoppered to allow staining for $3 \mathrm{~h}$ at $35^{\circ} \mathrm{C}$. Seed embryos were moistened with distilled water 3 times and $10 \mathrm{~mL}$ anhydrous ethanol added and placed at $35^{\circ} \mathrm{C}$ for $24 \mathrm{~h}$. Finally, the extraction solution was used to determine the OD value at $490 \mathrm{~nm}$. The test was conducted on three replicates.

\subsection{Field emergence test}

The field emergence test was conducted on three replicates of 50 seeds each for each treatment. Seeds were sown in Zhejiang and Beijing. The number of seedling was recorded after emergence until no emergence. FES was denoted by the number of days when $50 \%$ FEP was reached.

\subsection{Validation of vigor indices}

To validate the veracity of VA, DA as optimal index for evaluating seed vigor of super sweet corn and sugar enhanced corn, seeds of super sweet corn "Green Superman" and sugar enhanced corn "Zhongtian 2" were bought in 2005. A part of seeds were stored at $4^{\circ} \mathrm{C}$ and the others were stored at room temperature. VA of "Green Superman", DA of "Zhongtian 2" and their field performances were tested. 


\subsection{Q2 test and laboratory germination test}

Ten lots of super sweet corn "Green Superman" seeds were used for Q2 test. The Q2 test conducted on four replicates of 100 seeds per treatment quantified energy use by measuring oxygen consumption of individual seeds, planted in 16 microtiter plates of 96 wells with $200 \mu 1$ water and relating the oxygen consumption seed-by-seed to laboratory germination performances of the seeds. The Q2 oxygen measurement is temperature dependent because the measurement is based on partial oxygen pressure, which is temperature dependent. Thus, for sweet corn oxygen measurement, the temperature was adjusted to $20^{\circ} \mathrm{C}$. The test was performed at an interval of 30 min until $72 \mathrm{~h}$. These information was set by Q2 software program. The seeds were airproofed and oxygen content was measured by Q2 instrument. Five Q2 values including IMT, OMR, COP, RGT, HOM were calculated. The laboratory germination test was conducted on four replicates of 100 seeds per hybrid between two layers of filter paper. Seeds were incubated for 7 days at $20^{\circ} \mathrm{C}$ with alternating $12 \mathrm{~h}$ periods of darkness and light. Normal seedlings were judged according to the ISTA rule (ISTA, 2007) and the number of germinated seedlings was recorded at the 7th day. Germination speed was denoted by the number of germinated seedling days at the 4 th day.

\subsection{Statistics}

Variance analysis, correlation analysis and regression analysis were carried out with SAS V8.02. $\chi^{2}$ test was conducted according to the following formula: $\chi^{2}=\Sigma(\mathrm{AV}-\mathrm{PV}) 2 / \mathrm{PV}(\mathrm{AV}$ is for actual value and $\mathrm{PV}$ is for prediction value).

\section{RESULTS AND DISCUSSION}

\subsection{Evaluation indices for seed vigor of sweet corn}

It was seen from Table 1 that EC, SS, VA and DA of sweet corn were significantly correlated $(P \leq 0.01)$ with both FEP and FES. The results suggested that all the four indices were suitable for evaluating seed vigor of sweet corn.

It is known that there are different genetic backgrounds between super sweet corn (sh2) and sugar enhanced corn (se). Therefore, we considered that there might be some differences in evaluating seed vigor of super sweet corn and sugar enhanced corn. 
The correlation analysis results showed that EC $(P \leq 0.05)$, SS $(P \leq 0.05)$ and VA $(P \leq 0.01)$ significantly correlated with both FEP and FES. However, there was a higher correlation of VA than the former two indices. Therefore, VA was optimal index for evaluating seed vigor of super sweet corn.

The correlation analysis results showed that EC $(P \leq 0.05)$ and DA $(P \leq$ $0.01)$ significantly correlated with both FEP and FES. However, there was a higher correlation of DA than EC. Therefore, DA was optimal index for evaluating seed vigor of sugar enhanced corn.

Wang et al. (2004) considered EC was optimal index for evaluating seed vigor of legumes. Furthermore, it was also suitable for evaluating seed vigor of super sweet corn (Wilson et al., 1992), which was consistent to our results. In our results, SS could be used for evaluating seed vigor of super sweet corn, whereas it was unsuitable for evaluating seed vigor of sugar enhanced corn. The difference could be related with higher SS of super sweet corn than that of sugar enhanced corn. Our results showed that VA was an optimal index for evaluating seed vigor of super sweet corn while it was unsuitable for evaluating seed vigor of sugar enhanced corn, and DA was an optimal index for evaluating seed vigor of sugar enhanced corn while it was unsuitable for evaluating seed vigor of super sweet corn.

Table 1. Correlation coefficient between testing index and field performance of sweet corn.

\begin{tabular}{|c|c|c|c|c|c|c|}
\hline \multirow{2}{*}{ Testing indices } & \multicolumn{2}{|c|}{ Sweet corn $(n=36)$} & \multicolumn{2}{|c|}{ Super sweet corn $(n=18)$} & \multicolumn{2}{|c|}{ Sugar enhanced corn $(n=18)$} \\
\hline & FEP (\%) & FES (d) & FEP $(\%)$ & FES (d) & FEP $(\%)$ & FES (d) \\
\hline $\mathrm{EC}\left(\mu \Omega \cdot \mathrm{cm}^{-1} \cdot \mathrm{g}^{-1}\right)$ & $-0.678 * *$ & $0.543 * *$ & $-0.548^{*}$ & $0.571 *$ & $-0.519^{*}$ & $0.571^{*}$ \\
\hline $\mathrm{SS}\left(\mathrm{mg} \cdot \mathrm{g}^{-1}\right)$ & $-0.603 * *$ & $0.610^{* *}$ & $-0.533^{*}$ & $0.586^{*}$ & -0.447 & 0.421 \\
\hline $\mathrm{VA}(\mu \mathrm{g})$ & $-0.529 * *$ & $0.564 * *$ & $-0.713 * *$ & $0.725^{* *}$ & -0.408 & 0.203 \\
\hline $\mathrm{DA}\left(\mathrm{OD}_{490}\right)$ & $0.585^{* *}$ & $-0.566 * *$ & 0.368 & -0.214 & $0.702 * *$ & $-0.668 * *$ \\
\hline
\end{tabular}

\subsection{Validation for evaluation indices of seed vigor of sweet corn}

According to the above results for correlation analysis, regression equations were established by VA or DA as independent variable $\mathrm{x}$ and FEP or FES as dependent variable $y$. The regression equations of $y=-$ $16.074 x+86.355$ and $y=1.940 x+4.050$ were used to forecast FEP and FES of super sweet corn, respectively. The regression equations of $y=196.830 x+15.634$ and $y=-27.315 x+14.513$ were used to forecast FEP and FES of sugar enhanced corn, respectively.

It was seen from Table 2 that VA of "Green Superman" at room temperature was higher than at $4^{\circ} \mathrm{C}$, which was consistent to their vigor 
status. DA of "Zhongtian 2" at room temperature was lower than at $4{ }^{\circ} \mathrm{C}$, which was also consistent to their vigor status. To test the consistency of the actual value and the value of field emergence performance predicted by the above formulas, $\chi^{2}$ test was performed. $\chi^{2}$ test results showed that the four values of $\chi^{2}$ were all far lower than the value of $\chi_{0.05,1}^{2}$ (3.84), which suggested that the predicted value was consistent to the actual value. The verification experiment sufficiently indicated that VA and DA was very reliable for evaluating seed vigor of super sweet corn and sugar enhanced corn, respectively.

Table 2. The validation result of VA for seed evaluating seed vigor of super sweet corn and DA for evaluating seed vigor of sugar enhanced corn.

\begin{tabular}{lccccccccc}
\hline \multirow{2}{*}{ Hybrid } & \multirow{2}{*}{ Storage condition } & \multirow{2}{*}{$\mathrm{VA}(\mu \mathrm{g})$} & $\mathrm{DA}\left(\mathrm{OD}_{490}\right)$ & \multicolumn{3}{c}{ FEP $(\%)$} & \multicolumn{4}{c}{ FES $(\mathrm{d})$} \\
\cline { 5 - 10 } & & & & $\mathrm{AV}$ & $\mathrm{PV}$ & $\chi^{2}$ & $\mathrm{AV}$ & $\mathrm{PV}$ & $\chi^{2}$ \\
\hline \multirow{2}{*}{ Green } & $\mathrm{RM}$ & 2.566 & - & 48.0 & 45.1 & & 9.5 & 9.0 & \multirow{2}{*}{0.07} \\
\multirow{2}{*}{ Zhongerman } & $4^{\circ} \mathrm{C}$ & 1.995 & - & 52.0 & 54.3 & & 8.28 & 7.9 & \\
& $\mathrm{RM}$ & - & 0.151 & 42.0 & 45.2 & & 11.0 & 10.4 & 0.04 \\
\hline
\end{tabular}

FEP, field emergence percentage; FES, field emergence speed; RM, room temperature; $O D_{490}$, optical density at the wave length of $490 \mathrm{~nm}$; $A V$, actual value; $P V$, prediction value; $\chi_{0.05,1}^{2}=3.84$.

\subsection{The application of Q2 oxygen sensing technology to seed vigor testing}

It was seen from Table 3 that OMR of super sweet corn "Green Superman" was positively correlated with both LGP $(P \leq 0.01)$ and LGS $(P$ $\leq 0.05)$. Furthermore, COP was negatively correlated with both LGP $(P \leq$ $0.05)$ and LGS $(P \leq 0.01)$. However, the other three Q2 values were not significantly correlated with LGP or LGS. The results suggested that OMR and COP were suitable for evaluating seed vigor of super sweet corn "Green Superman". The above results were the initial ones, which need further study and validation.

Table 3. Correlation coefficient between Q2 value and laboratory germination performance of super sweet corn "Green Superman".

\begin{tabular}{lcc}
\hline Q2 value & LGP $(\%)$ & LGS $(\%)$ \\
\hline IMT $(\mathrm{h})$ & -0.321 & -0.339 \\
OMR $\left(\% \cdot \mathrm{h}^{-1}\right)$ & $0.582^{* *}$ & $0.549^{*}$ \\
COP $(\%)$ & $-0.487^{*}$ & $-0.570^{* *}$ \\
RGT $\left(\mathrm{h}^{-1}\right)$ & 0.395 & 0.264 \\
HOM $\left(\mathrm{h}^{-2}\right)$ & 0.213 & 0.378 \\
\hline
\end{tabular}

$*, * *$ denote significant correlation at $P \leq 0.05$ and $P \leq 0.01$, respectively. $L G P$, laboratory germination percentage; LGS, laboratory germination speed; IMT, increased metabolism time; OMR, oxygen metabolism rate; COP, critical oxygen pressure; $R G T$, relative germination time; HOM, homogeneity, or uniformity of the RGT. $r_{0.05,18}=0.444, r_{0.01,18}=0.561$. 

Corn

\section{CONCLUSIONS AND FUTURE WORKS}

In conclusion, EC, SS, VA, DA, OMR and COP were all suitable to evaluate seed vigor of sweet corn. Furthermore, VA was optimal for evaluating seed vigor of super sweet corn. DA was optimal for evaluating sugar enhanced corn.

For Q2 test, however, laboratory germination performances were used as the vigor indices, which was lack of stringency to response the vigor status. Furthermore, only super sweet corn "Green Superman" was used. Therefore, to validate the reliability of OMR and COP, in the future works, field test will be conducted and other sweet corn hybrids will be used for Q2 test.

\section{ACKNOWLEDGEMENTS}

We give our thanks to Johan van Asbrouck, vice president of research \& technology and Freek Schreurs, director of new business, ASTEC Global, Netherlands for Q2 oxygen sensing technology information.

\section{REFERENCES}

Chen Ruizheng, Zhang Hongwei, Fu Jiarui, et al.. Studies on the association of rice seed vigor witth volatile aldehydes, Acta Scientiarum Naturalium Universitatis Sunyatseni, 1996, 35 : 54-57 (in Chinese)

D. O. J. Wilson, J. C. Alleyne, B. Shafii, et al. Combining vigour test results for prediction of final stand of shrunken-2 sweet corn seed, Crop Science, 1992, 32: 1496-1502

International Seed Testing Association (ISTA). International rules for seed testing edition 2007

J. M. Woltz, D. M. TeKrony. Accelerated ageing test for corn seed, Seed Technology, 2001, 23: $21-34$

L. J. Waters, B. L. Blanchette. Prediction of sweet corn field emergence by conductivity and cold tests, Journal American Society for Horticultural Science, 1983, 108: 778-781

M. Akram, M. Munir, S. U. Ajmal, et al. Seed and seedling vigour in rice: Association among the traits, Pakistan Journal of Seed Technology, 2002, 1: 25-30

Qiao Yanxiang, Gao Pingping, Ma Junhua, et al.. Study on physiological characteristics and seed activity changes of two maize inbreds in ageing course, Acta Agronomica Sinica, 2003, 29: $123-127$

S. O. J. Wilson, M. B. J. McDonald. A convenient volatile aldehyde assay for measuring soybean seed vigour, Seed Science and Technology, 1986, 14: 259-268

T. M. Ching, S. Hedtke, M. C. Boulger, et al. Correlation of field emergence rate and seed vigor criteria in barley cultivars, Crop Science, 1977, 17: 312-314

Tao Jialing, Zheng Guanghua. Seed vigor. Beijing: Science Press, 1991 (in Chinese)

Y. R. Wang, L. Yu, Z. B. Nan, et al. Vigour tests used to rank seed lot quality and predict field emergence in four forage species, Crop Science, 2004, 44: 535-541 
Zhang Shourui. Preliminary study on corn seed vigour testing and relationship between it and field emergence percentage, Gansu Agricultural Science and Technology, 1997, 2: 13-15 (in Chinese)

Zhao Guangwu, Sun Qun, Wang Jianhua. Improving seed vigour assessment of super sweet corn and sugar enhanced sweet corn, New Zealand Journal of Crop and Horticultural Science, 2007, 35(3): 349-356

Zhao Guangwu, Wang Jianhua. Seed vigor testing of sweet corn and evaluation of its field establishment ability, Plant Physiology Communications, 2005, 41: 444-448 (in Chinese)

Zhu Cheng, Zeng Guangwen. A convenient method for measuring the volatile aldehydes released from seed, Plant Physiology Communications, 1999, 35: 39-40 (in Chinese) 